\title{
Avaliação populacional de lagartas e inimigos naturais em azevém, com rede de varredura(1)
}

\begin{abstract}
Alexandre Specht ${ }^{(2)}$ e Elio Corseuil(3)
Resumo - Os noctuídeos constituem as principais pragas fitófagas de gramíneas e decisões sobre seu controle baseiam-se em porcentuais de desfolhamento, desconsiderando o grau de incidência da praga. Este estudo objetivou testar a rede de varredura na avaliação das populações de Noctuidae e principais grupos de inimigos naturais, em azevém (Lolium multiflorum Lam.), nos turnos da manhã e da noite. O trabalho foi conduzido em duas lavouras, em Salvador do Sul, RS. Realizou-se um experimento com coletas semanais e outro, complementar, com coletas em cada fase de desenvolvimento do azevém, mantendo criação laboratorial. No primeiro, foram coletadas 2.044 lagartas, das quais somente 151 foram coletadas no período matinal; à noite foram detectadas diferenças estatísticas entre datas de coleta e tamanho das lagartas. Também foram capturadas 387 aranhas, 577 microimenópteros e 983 outros insetos de interesse no controle biológico; houve significância estatística entre turnos e datas em relação a Carabidae, Forficulidae, Tachinidae, Nabidae, Vespidae, microimenópteros e Araneae. Do segundo experimento, obtiveram-se 11 espécies, das quais Pseudaletia sequax Franc., 1951, foi a predominante. $\mathrm{O}$ emprego da rede de varredura permite avaliar as populações de Noctuidae e principais grupos de inimigos naturais, em azevém, no turno da noite.
\end{abstract}

Termos para indexação: Lolium multiflorum, Noctuidae, população de insetos, manejo integrado de pragas.

\section{Evaluation of caterpillar populations and natural enemies in the ryegrass with sweeping net}

\begin{abstract}
Noctuids are the main phytophagous pests of gramineae and decisions about its control are based on percentage of defoliation, not considering the level of larval infestation. The objective of this study was to test the sweeping net in the evaluation Noctuidae population and of the principal groups of natural enemies in ryegrass (Lolium multiflorum Lam.), in the morning and at night. The work was conducted in two crops in Salvador do Sul, RS, Brazil. In the first experiment, insects were sampled weekly, and in the second one, which was complementary, the collections were done in each phase of ryegrass development, maintaining laboratorial rearing. From the first one, a total of 2,044 caterpillars were collected, but only 151 in the morning. Statistical differences occurred at night between sampling dates and caterpillar sizes. Three hundred and eighty seven spiders, 577 microhymenopterans and 983 other insects with interest in biological control were also captured; statistical significance was observed between day periods and dates for Carabidae, Forficulidae, Tachinidae, Nabidae, Vespidae, microhymenopterans and Araneae. From the second experiment eleven species were obtained, and Pseudaletia sequax Franc., 1951, was the predominant. The use of sweeping net allows the evaluation of Noctuidae population, as well as the main groups of natural enemies in ryegrass, in the evening.
\end{abstract}

Index terms: Lolium multiflorum, Noctuidae, insect population, integrated pest management.

(1) Aceito para publicação em 11 de abril de 2001.

Extraído da Dissertação de Mestrado apresentada pelo primeiro autor à Pontifícia Universidade Católica do Rio Grande do Sul, Porto Alegre, RS. Parcialmente financiado pelo CNPq.

(2)Universidade do Sul de Santa Catarina, Centro de Ciências Exatas, Agrárias e Engenharias, Caixa Postal 370, CEP 88704-900 Tubarão, SC. E-mail: spechta@terra.com.br

(3) Pontifícia Universidade Católica do Rio Grande do Sul, Faculdade de Biociências, Caixa Postal 1429, CEP 90619-900 Porto Alegre, RS. E-mail: corseuil@pucrs.br

\section{Introdução}

O azevém (Lolium multiflorum Lam.) é uma gramínea anual que se desenvolve em grande parte da região neotropical, especialmente onde predominam os climas temperado e frio (Burkart, 1975). Cresce em áreas cultivadas e em terrenos baldios, dominando o espaço em virtude da produção de aleloquímicos. É a principal forrageira de inverno- 
primavera e a mais expressiva cultura para produção de feno.

Entre os fatores limitantes das culturas de gramíneas estão os danos causados por insetos da ordem Lepidoptera, que muitas vezes caracterizamse como pragas-chave, por causa da facilidade de deslocamento e alta fecundidade (Tscharntke \& Greiler, 1995), apresentando atividade predominantemente noturna (Gassen, 1984; Gallo et al., 1988). No Brasil existem 30 espécies da família Noctuidae, hóspedes de pelo menos 20 gramíneas cultivadas (Silva et al., 1968).

Em soja vem sendo empregado um método simples e prático para avaliar as populações de pragas e inimigos naturais, que consiste em determinar os níveis populacionais que exigem controle. Tais monitoramentos propiciam a redução de gastos e de efeitos adversos ao homem e ao meio ambiente, pois permitem a utilização de medidas menos agressivas e mais específicas, como o controle biológico.

Os níveis populacionais de controle das pragas, em relação a Noctuidae, nas culturas de gramíneas, como o arroz, trigo e forrageiras, limitam-se a porcentuais de desfolhamento e a simples observação sobre a presença ou ausência de lagartas (Lima \& Racca Filho, 1996). No entanto, tais fatos suscitam problemas de ordem econômica e ambiental, motivados pela contaminação do ambiente e gastos desnecessários com produtos fitossanitários. Esses problemas acontecem quando, apesar da ocorrência de desfolhamento, as populações de insetos já diminuíram naturalmente, quando as espécies que estão atuando como pragas-chave ou ocasionais não são identificadas e quando há diminuição de agentes de controle biológico.

O objetivo deste estudo foi testar a rede de varredura para avaliar as populações de Noctuidae e principais grupos de inimigos naturais, em azevém, a partir de amostragens matinais e noturnas.

\section{Material e Métodos}

Este trabalho constou de dois experimentos, com delineamento completamente casualizado, conduzidos em duas lavouras de azevém, cada uma com aproximadamente
1,5 ha, em Salvador do Sul, RS, em altitude entre 460 e $470 \mathrm{~m}$, consideradas repetições.

A coleta dos insetos foi realizada por meio de rede de varredura com diâmetro de $40 \mathrm{~cm}$, acionada a partir das $10 \mathrm{~h}$ e $22 \mathrm{~h}$, referidos como turnos da manhã e da noite.

No primeiro experimento foram realizadas 17 coletas semanais, iniciadas em 22 de julho de 1994; as unidades amostrais constaram do somatório de cinco conjuntos de dez redadas em movimentos pendulares, com amplitude de $1 \mathrm{~m}$, extraídos de linhas de $2 \mathrm{~m}$ demarcadas em áreas de 15 x 20 m, evitando sobreposições subseqüentes. Em laboratório, as lagartas foram contadas, considerando-se pequenas as que mediam até $1,5 \mathrm{~cm}$; médias de $1,5 \mathrm{~cm}$ até $2,5 \mathrm{~cm}$; e grandes quando eram maiores do que $2,5 \mathrm{~cm}$. A maioria dos inimigos naturais foi identificada até nível de família.

No segundo experimento, foram efetuadas amostragens complementares, nas fases vegetativa, reprodutiva e de maturação do azevém, para criação laboratorial das lagartas visando à identificação precisa a partir das formas adultas. Para tanto, cada coleta teve duas repetições de 150 redadas, por turno, em áreas adjacentes ao primeiro ensaio.

As variações populacionais foram avaliadas por meio de análises de variância, cujos dados foram transformados em $(x+0,5)^{0,5}$, sendo suas médias agrupadas pelo teste de amplitude múltipla de Duncan a 5\% de probabilidade. Os números de lagartas foram analisados como um trifatorial, com turno, tamanho e data de coleta; a variação do número de indivíduos de espécies entomófagas, por um bifatorial com turno e data de coleta, e os adultos provenientes das coletas complementares, por um bifatorial com as fases de desenvolvimento do azevém e turno. As coletas da primeira e da última semana de outubro foram prejudicadas pela elevada precipitação e fortes ventos, deixando de ser consideradas.

\section{Resultados e Discussão}

No primeiro experimento foram obtidas 2.044 lagartas, das quais $151(7,4 \%)$ foram coletadas durante o dia (Tabela 1). O maior número de lagartas coletadas à noite é atribuído ao fato de os noctuídeos, como as demais mariposas, apresentarem hábitos noturnos ou crepusculares, também referido em re- 
lação às formas jovens, as quais procuram, durante o dia, abrigo sob as folhas ou no solo (Gassen, 1984; Gallo et al., 1988).

As maiores variações populacionais ocorreram entre as coletas de 24 de setembro e sete de novembro, com grandes diferenças entre os turnos de coleta e tamanho das lagartas. As coletas matinais apresentaram variação entre semanas em relação às lagartas pequenas e médias. Apesar de as lagartas pequenas apresentarem maior número em 25 de outubro, não diferiram significativamente das demais datas de coleta. As lagartas médias também apresentaram maior número nessa mesma data e diferiram significativamente apenas das datas de 31 de maio e sete de agosto. Nas coletas noturnas, com números médios bastante elevados, o pico populacional das lagartas de todos os tamanhos ocorreu nas três datas de outubro, com alguma igualdade estatística entre as de tamanho médio e grande. A ocorrência de lagartas pequenas em maior número, não seguidas pelos demais tamanhos, especialmente à noite, é indício de alta mortalidade nos primeiros ínstares, o que pode ser causado pelas condições climáticas adversas e, especialmente, pela ação de inimigos naturais.

Esses resultados evidenciam que a utilização noturna da rede de varredura proporciona boa avalia- ção populacional de lagartas, cujos números, em razão dos diferentes tamanhos, poderão contribuir para futuros estudos de níveis de dano econômico em gramíneas e decisões sobre uso de microrganismos ou produtos seletivos dentro do manejo integrado de pragas (MIP). Sugere-se antecipar o horário noturno para maior facilidade na varredura e para evitar eventual orvalho.

No segundo experimento, foram obtidas 523 lagartas das quais emergiram adultos de Anicla ignicans (Guenée, 1852), Anicla infecta (Ochsenheimer, 1816), Dargida meridionalis (Hampson, 1905), Faronta albilinea (Hübner, 1827), Leucania humidicola Guenée, 1852, Peridroma saucia (Hübner, 1808), Pseudaletia sequax Franclemont, 1951, Pseudoleucania butleri (Schaus, 1898), Spodoptera eridania (Stoll, 1782), Spodoptera frugiperda (J.E. Smith, 1797) e Spodoptera latifascia (Walker, 1856). Os números médios das espécies mais expressivas de tais noctuídeos, por fase de desenvolvimento, estão representados na Tabela 2.

A espécie preponderante, $P$. sequax, ocorreu em menor número na fase vegetativa sendo mais coletada à noite, com acme populacional na fase reprodutiva. Por ser a mais abundante, atribuem-se a

Tabela 1. Número médio de lagartas de noctuídeos de diferentes tamanhos, coletadas com rede de varredura, em azevém, de acordo com o turno e a data. Salvador do Sul, RS, 1994(1).

\begin{tabular}{|c|c|c|c|c|c|c|}
\hline \multirow[t]{2}{*}{ Data } & \multicolumn{3}{|c|}{ Coletas matinais } & \multicolumn{3}{|c|}{ Coletas noturnas } \\
\hline & Pequenas & Médias & Grandes & Pequenas & Médias & Grandes \\
\hline $31 / 7$ & $0,50 \mathrm{ab}$ & $0,00 \mathrm{~b}$ & 1,00 & $0,50 \mathrm{~cd}$ & $4,50 \mathrm{de}$ & $1,00 \mathrm{c}$ \\
\hline $7 / 8$ & $1,00 \mathrm{ab}$ & $0,00 \mathrm{~b}$ & 0,00 & $0,50 \mathrm{~cd}$ & $1,00 \mathrm{e}$ & $0,50 \mathrm{c}$ \\
\hline $13 / 8$ & $3,00 \mathrm{ab}$ & $1,00 \mathrm{ab}$ & 0,00 & $2,00 \mathrm{~cd}$ & $2,50 \mathrm{de}$ & $1,00 \mathrm{c}$ \\
\hline $21 / 8$ & $2,00 \mathrm{ab}$ & $1,00 \mathrm{ab}$ & 0,50 & $1,00 \mathrm{~cd}$ & $3,50 \mathrm{de}$ & $1,00 \mathrm{c}$ \\
\hline $27 / 8$ & $1,50 \mathrm{ab}$ & $0,00 \mathrm{~b}$ & 0,00 & $0,00 \mathrm{~d}$ & $0,00 \mathrm{e}$ & $0,00 \mathrm{c}$ \\
\hline $3 / 9$ & $6,50 \mathrm{ab}$ & $6,00 \mathrm{ab}$ & 0,50 & $1,00 \mathrm{~cd}$ & $4,00 \mathrm{cde}$ & $0,50 \mathrm{c}$ \\
\hline $10 / 9$ & $3,50 \mathrm{ab}$ & $0,00 b^{(2)}$ & 0,00 & $5,50 \mathrm{~cd}$ & $6,00 \mathrm{cde}$ & $2,50 \mathrm{c}$ \\
\hline $18 / 9$ & $1,50 \mathrm{ab}$ & $1,00 \mathrm{ab}$ & 0,00 & $7,50 \mathrm{c}$ & $5,00 \mathrm{cde}$ & $2,50 \mathrm{c}$ \\
\hline $24 / 9$ & $6,50 \mathrm{ab}^{(2)}$ & $1,00 \mathrm{ab}^{(2)}$ & 0,50 & $48,00 \mathrm{bA}$ & $10,00 \mathrm{cdB}$ & $3,00 \mathrm{cB}$ \\
\hline $8 / 10$ & $5,00 \mathrm{ab}^{(2)}$ & $3,00 \mathrm{ab}^{(2)}$ & $0,00^{(2)}$ & $176,50 \mathrm{aA}$ & $63,50 \mathrm{aB}$ & $34,00 \mathrm{abC}$ \\
\hline $16 / 10$ & $6,50 a b^{(2)}$ & $0,50 a b^{(2)}$ & $0,50^{(2)}$ & $173,50 \mathrm{aA}$ & $38,00 \mathrm{bB}$ & $54,00 \mathrm{aB}$ \\
\hline $25 / 10$ & $8,50 \mathrm{aA}^{(2)}$ & $8,00 \mathrm{aA}^{(2)}$ & $0,50 \mathrm{~B}^{(2)}$ & $156,50 \mathrm{aA}$ & $51,50 \mathrm{abB}$ & $35,50 \mathrm{abB}$ \\
\hline $7 / 11$ & $0,00 b^{(2)}$ & $0,50 a b^{(2)}$ & $0,00^{(2)}$ & $12,00 \mathrm{c}$ & $14,50 \mathrm{c}$ & $19,00 \mathrm{~b}$ \\
\hline $14 / 11$ & $0,00 \mathrm{~b}$ & $0,00 \mathrm{~b}$ & 0,00 & $4,00 \mathrm{~cd}$ & $4,00 \mathrm{cde}$ & $1,50 \mathrm{c}$ \\
\hline $20 / 11$ & $0,50 \mathrm{ab}$ & $0,00 \mathrm{~b}$ & 0,00 & $2,00 \mathrm{~cd}$ & $3,00 \mathrm{~cd}$ & $1,00 \mathrm{c}$ \\
\hline
\end{tabular}

(1)Médias seguidas por letras distintas, minúscula na coluna e maiúscula na linha, diferem significativamente entre si pelo teste de Duncan a $5 \%$ de probabilidade; análise com valores transformados em raiz quadrada de $(x+0,5){ }^{(2)}$ Indicam as diferenças entre turno para cada tamanho na respectiva data de coleta. 
ela as maiores diferenças dos números de lagartas coletadas entre 24 de setembro e sete de novembro (Tabela 1). Comportamento diferente foi observado em A. ignicans e D. meridionalis, que tiveram os maiores números na fase vegetativa, não havendo diferença significativa entre turnos, e $S$. frugiperda, que teve um aumento populacional apenas na última fase (Tabela 2). Os resultados indicam que, também nesta cultura, a comunidade de Noctuidae é composta por uma praga-chave, $P$. sequax, que representou $78 \%$, e outras menos expressivas, destacando-se D. meridionalis (11\%), A. ignicans (6\%) e $S$. frugiperda $(2 \%)$. As causas que determinaram as variações dos níveis populacionais observados nas diferentes espécies, em razão das épocas ou fases de desenvolvimento do azevém, devem ser objeto de futuras investigações.

Em relação à abundância dos organismos relacionados com o controle natural, presentes na área do experimento, foram coletados 387 aranhas, 577 microimenópteros e 983 outros insetos distribuídos em oito ordens e 19 famílias (Tabela 3).

Os carabídeos foram capturados em maior número à noite, ocorrendo um pico populacional dia 25 de outubro (Tabela 4). As espécies capturadas de dia foram, na maioria, de pequeno porte, especialmente Callida sp., Gallerita colaris e Lebia concinna. O comportamento diferenciado quanto ao turno de atividade e tamanho das espécies foi coerente com as observações de Luff (1978). Cabe ressaltar que pelo hábito de muitas espécies andarem junto ao solo, as amostragens com rede de varredura subestimam as populações.

O número de dermápteros amostrados só foi expressivo à noite (Tabela 4), confirmando as indicações sobre hábito noturno de Lima (1939), Costa (1958) e Gallo et al. (1988). Observou-se uma elevação populacional a partir de 16 de outubro, com acme nos dias sete e 14 de novembro. $\mathrm{O}$ fato de a população ter os maiores incrementos após o acme populacional das lagartas (Tabela 1), mantendo-se até o final da cultura, evidencia a relação predador-presa, salientada pelos estudos sobre a capacidade predatória de lagartas (Reis et al., 1988) e ovos (Cruz et al., 1995).

$\mathrm{O}$ número de taquinídeos coletados não diferiu significativamente entre os turnos, cujo número de indivíduos amostrados foi mais expressivo nas últimas coletas (Tabela 4). O fato de serem coletados em ambos os turnos evidencia sua permanência na cultura durante a noite. Nas lagartas mantidas em criação, o nível de parasitismo foi de 4,7\%.

Os nabídeos foram significativamente mais coletados à noite $(1,57 \mathrm{a})$ do que pela manhã $(0,47 \mathrm{~b})$, porém não houve diferença entre as coletas. Apesar de serem predadores generalistas, capazes de exercer pressão sobre populações de lagartas (Lima, 1940; Corrêa-Ferreira \& Moscardi, 1985; Gassen, 1986), não foi possível observar um desenvolvimento proporcional com o da população de lagartas.

Tabela 2. Número médio de noctuídeos adultos obtidos de criação a partir de lagartas coletadas nas diferentes fases de desenvolvimento do azevém, em dois turnos de coletas. Salvador do Sul, RS, 1994(1).

\begin{tabular}{llccc}
\hline Espécie & Turno & \multicolumn{2}{c}{ Fase de desenvolvimento do azevém } \\
\cline { 2 - 5 } & & Vegetativa & Reprodutiva & Maturação \\
\hline Pseudaletia sequax & Diurno & $4,50 \mathrm{bB}$ & $13,50 \mathrm{aB}$ & $10,00 \mathrm{aB}$ \\
& Noturno & $10,50 \mathrm{cA}$ & $115,50 \mathrm{aA}$ & $52,50 \mathrm{bA}$ \\
\hline Dargida meridionalis & Diurno & 10,50 & 3,00 & 4,50 \\
& Noturno & 5,50 & 1,50 & 4,50 \\
\hline Anicla ignicans & Diurno & 4,50 & 1,50 & 2,50 \\
& Noturno & 3,00 & 0,00 & 1,50 \\
\hline Spodoptera frugiperda & Diurno & 0,00 & 0,50 & 1,00 \\
& Noturno & 0,50 & 3,00 \\
\hline
\end{tabular}

${ }^{(1)}$ Médias seguidas por letras distintas, minúscula na linha e maiúscula na coluna, diferem significativamente entre si pelo teste de Duncan a $5 \%$ de probabilidade; análise com valores transformados em raiz quadrada de $(\mathrm{x}+0,5)$. 
Os vespídeos foram amostrados em maior número de dia, especialmente nas últimas coletas, não revelando diferenças à noite (Tabela 4). A maioria das vespas capturadas foi Polybia scutellaris (White), e o fato de serem coletadas em pequeno número pode estar relacionado com as baixas temperaturas e pre-

Tabela 3. Insetos relacionados ao controle biológico de noctuídeos coletados com rede de varredura, em azevém. Salvador do Sul, RS, 1994.

\begin{tabular}{llr}
\hline Ordem & Família & Número \\
\hline Coleoptera & Carabidae & 56 \\
& Coccinellidae & 47 \\
Dermaptera & Staphylinidae & 22 \\
Diptera & Forficulidae & 315 \\
& Asilidae & 11 \\
Hemiptera & Tachinidae & 203 \\
& Anthocoridae & 1 \\
& Nabidae & 67 \\
& Pentatomidae & 2 \\
Hymenoptera & Reduviidae & 28 \\
& Braconidae & 28 \\
& Ichneumonidae & 4 \\
Mantodea & Vespidae & 112 \\
Neuroptera & Vatidae & 5 \\
& Chrysopidae & 27 \\
Odonata & Hemerobiidae & 3 \\
& Calopterygidae & 1 \\
& Coenagrionidae & 42 \\
& Libellulidae & 9 \\
\hline
\end{tabular}

sença de orvalho, pela manhã, e, à noite, em razão dos reduzidos números de exemplares que eventualmente permaneciam em campo. Tais resultados reforçam a indicação de que a maior atividade das vespas ocorre nas horas mais quentes do dia (Giannotti et al., 1995).

Os microimenópteros foram capturados em maior número de manhã, com um pico populacional dia 10 de setembro, não sendo observadas diferenças entre coletas noturnas (Tabela 4). Foram responsáveis pelo parasitismo de $8,67 \%$ das lagartas mantidas em criação, representados principalmente por espécies de Apanteles e Euplectrus. Apesar da sua importância no controle biológico dos lepidópteros (Lima, 1962; Silva et al., 1968; Gassen, 1986), maiores relações entre estes parasitóides e as lagartas coletadas dependem de identificação específica, uma vez que podem ter outros hospedeiros.

Apesar da importância no controle biológico de pragas, os demais grupos de insetos representaram apenas $14,7 \%$, e são predadores generalistas, dificultando a compreensão do seu papel no controle natural (Tabela 3).

As aranhas foram capturadas em maior número à noite, com pico populacional dia 13 de agosto, com alguma igualdade estatística em várias outras datas

Tabela 4. Médias dos inimigos naturais de Noctuidae, coletados com rede de varredura, em azevém, de acordo com as datas e turnos. Salvador do Sul, RS, 1994(1).

\begin{tabular}{|c|c|c|c|c|c|c|}
\hline \multirow[t]{3}{*}{ Data } & \multirow{3}{*}{$\begin{array}{c}\text { Coleoptera: } \\
\text { Carabidae }\end{array}$} & \multirow{3}{*}{$\begin{array}{c}\text { Diptera: } \\
\text { Tachinidae }\end{array}$} & \multirow[t]{3}{*}{ Araneae } & \multirow{3}{*}{$\begin{array}{c}\text { Dermaptera: } \\
\text { Forficulidae } \\
\text { Noturno }\end{array}$} & \multicolumn{2}{|c|}{ Hymenoptera } \\
\hline & & & & & Vespidae & Microimenópteros \\
\hline & & & & & \multicolumn{2}{|c|}{ Diurno } \\
\hline $31 / 7$ & $0,75 b$ & $0,00 \mathrm{~b}$ & $6,75 \mathrm{ab}$ & $1,50 \mathrm{def}$ & $0,00 \mathrm{e}$ & $16,00 \mathrm{bc}^{(2)}$ \\
\hline $7 / 8$ & $0,00 \mathrm{~b}$ & $0,25 b$ & $4,25 \mathrm{abc}$ & $0,00 \mathrm{f}$ & $0,00 \mathrm{e}$ & $11,50 \mathrm{bcd}$ \\
\hline $13 / 8$ & $0,50 \mathrm{~b}$ & $0,00 \mathrm{~b}$ & $11,75 \mathrm{a}$ & $2,00 \mathrm{def}$ & $0,00 \mathrm{e}$ & $27,00 b^{(2)}$ \\
\hline $21 / 8$ & $0,00 \mathrm{~b}$ & $0,00 \mathrm{~b}$ & $5,75 \mathrm{ab}$ & $0,50 \mathrm{ef}$ & $0,00 \mathrm{e}$ & $26,00 b^{(2)}$ \\
\hline $10 / 9$ & $0,00 \mathrm{~b}$ & $0,25 b$ & $5,25 \mathrm{abc}$ & $6,50 \mathrm{cde}^{(2)}$ & $1,50 \mathrm{de}$ & $56,50 \mathrm{a}^{(2)}$ \\
\hline $18 / 9$ & $0,25 b$ & $0,25 b$ & $6,25 \mathrm{ab}$ & $5,50 \mathrm{cdef}^{(2)}$ & $2,00 \mathrm{cde}^{(2)}$ & $16,00 \mathrm{bc}$ \\
\hline $24 / 9$ & $0,00 \mathrm{~b}$ & $0,25 b$ & $5,75 \mathrm{ab}$ & $2,00 \mathrm{def}$ & $0,00 \mathrm{e}$ & $0,50 \mathrm{e}$ \\
\hline $8 / 10$ & $0,75 b$ & $3,50 \mathrm{ab}$ & $10,00 \mathrm{ab}$ & $8,50 \mathrm{bcd}^{(2)}$ & $2,50 \mathrm{~cd}^{(2)}$ & $25,00 b^{(2)}$ \\
\hline $16 / 10$ & $0,75 b$ & $2,00 \mathrm{ab}$ & $8,50 \mathrm{ab}$ & $21,00 \mathrm{ab}^{(2)}$ & $7,00 b^{(2)}$ & $6,50 \mathrm{cde}$ \\
\hline Diurno & $0,13 b$ & $3,73 a$ & $4,10 b$ & $0,30 \mathrm{~b}$ & $2,73 a$ & $14,53 \mathrm{a}$ \\
\hline Noturno & $1,43 \mathrm{a}$ & $2,50 \mathrm{a}$ & $8,67 \mathrm{a}$ & $9,23 \mathrm{a}$ & $0,23 b$ & $3,90 \mathrm{~b}$ \\
\hline
\end{tabular}

${ }^{(1)}$ Médias seguidas por letras distintas, em cada coluna, diferem significativamente entre si pelo teste de Duncan a $5 \%$ de probabilidade; análise com valores transformados em raiz quadrada de $(x+0,5)$. ${ }^{(2)}$ Indicam as diferenças entre turnos para cada grupo de inimigo natural na respectiva data de coleta. 
(Tabela 4). Representaram 20,8\% dos organismos entomófagos avaliados, o que destaca sua importância como já referido por Schoenly (1990).

O emprego da rede de varredura, à noite, poderá auxiliar nas decisões sobre controle de lagartas em gramíneas. No entanto, devem ser intensificados estudos referentes à avaliação das populações de insetos em culturas de maior interesse econômico e experimentação com outros intervalos entre coletas e horários.

\section{Conclusões}

1. O emprego da rede de varredura permite avaliar as populações de Noctuidae, com melhores resultados em coletas noturnas do que matutinas.

2. Os melhores turnos para avaliação de inimigos naturais variam em razão dos principais grupos.

\section{Referências}

BURKART, A. Evolution of grasses and grasslands in South America. Taxon, Berlin, v. 24, n. 1, p. 53-66, 1975.

CORRÊA-FERREIRA, B. S.; MOSCARDI, F. Potencial de consumo dos principais insetos predadores ocorrentes na cultura da soja. In: EMBRAPA. Centro Nacional de Pesquisa de Soja (Londrina, PR). Resultados de pesquisa de soja: 1984/85. Londrina: Embrapa-CNPSo, 1985. p. 79. (Documentos, 15).

COSTA, R. G. Alguns insetos e outros pequenos animais que danificam plantas cultivadas no Rio Grande do Sul. Porto Alegre: Secretaria da Agricultura, Indústria e Comércio, 1958. 296 p. (SIPA, 172. Série A).

CRUZ, I.; ALVARENGA, C. D.; FIGUEIREDO, P .E. F. Biologia de Doru luteipes (Scudder) e sua capacidade predatória de ovos de Helicoverpa zea (Boddie). Anais da Sociedade Entomológica do Brasil, Londrina, v. 24, n. 2, p. 273-278, 1995.

GALLO, D.; NAKANO, O.; SILVEIRA NETO, S.; CARVALHO, R. P. L.; BATISTA, G. C. de; BERTI FILHO, E.; PARRA, J. R. P.; ZUCCHI, R. A.; ALVES, S. B.; VENDRAMIM, J. D. Manual de entomologia agrícola. São Paulo: Ceres, 1988. 649 p.
GASSEN, D. N. Insetos associados à cultura do trigo no Brasil. Passo Fundo: Embrapa-CNPT, 1984. 39 p. (Circular Técnica, 3).

GASSEN, D. N. Parasitos, patógenos e predadores de insetos associados à cultura do trigo. Passo Fundo: Embrapa-CNPT, 1986. 86 p. (Circular Técnica, 1).

GIANNOTTI, E.; PREZOTO, F.; MACHADO, V. L. L. Foraging activity of Polistes lanio lanio (Fabr.) (Hymenoptera: Vespidae). Anais da Sociedade Entomológica do Brasil, Londrina, v. 24, n. 3, p. 455-463, 1995.

LIMA, A. F.; RACCA FILHO, F. Manual de pragas e praguicidas: receituário agronômico. Rio de Janeiro: Ed. da UFRRJ, 1996. 818 p.

LIMA, A. M. da C. Hemípteros. In: Insetos do Brasil. Rio de Janeiro: Imprensa Nacional, 1940. t. 2, cap. 22. (Escola Nacional de Agronomia. Série Didática, 3).

LIMA, A. M. da C. Himenópteros: $2^{a}$ parte. In: Insetos do Brasil. Rio de Janeiro: Imprensa Nacional, 1962. t. 12, cap. 30. (Escola Nacional de Agronomia. Série Didática, 14).

LIMA, A. M. da C. Insetos do Brasil. Rio de Janeiro: Imprensa Nacional, 1939. t. 1. (Escola Nacional de Agronomia. Série Didática, 2).

LUFF, M. L. Diel activity patterns of some field Carabidae. Ecological Entomology, Torquay, v. 3, n. 1, p. 53-62, 1978.

REIS, L. L.; OLIVEIRA, L. J.; CRUZ, I. Biologia e potencial de Doru luteipes no controle de Spodoptera frugiperda. Pesquisa Agropecuária Brasileira, Brasília, v. 23, n. 4, p. 333-342, abr. 1988.

SCHOENLY, K. The predators of insects. Ecological Entomology, Torquay, v. 15, n. 1, p. 333-345, 1990.

SILVA, A. G. A.; GONÇALVES, C. R.; GALVÃO, D. M.; GONÇALVES, A. J. L.; GOMES, J.; SILVA, M. do N.; SIMONI, L. de. Insetos, hospedeiros e inimigos naturais. In: . Quarto catálogo dos insetos que vivem nas plantas do Brasil: seus parasitos e predadores. Rio de Janeiro: Ministério de Agricultura, 1968. t. 1, pt. 2.

TSCHARNTKE, T.; GREILER, H. J. Insect communities, grasses, and grasslands. Annual Review of Entomology, Palo Alto, v. 40, p. 535-558, 1995. 